PROFESSOR AMANDA J NICOLL (Orcid ID : 0000-0002-1855-2757)

PROFESSOR STUART KEITH ROBERTS (Orcid ID : 0000-0002-9015-7997)

DR JACOB GEORGE (Orcid ID : 0000-0003-3716-7683)

DR EDMUND TSE (Orcid ID : 0000-0003-0643-3926)

Article type : Original Scientific Paper

\title{
BENEFICIAL RESPONSE TO MYCOPHENOLATE MOFETIL BY PATENTS WITH AUTOIMMUNE HEPATITIS, WHO HAVE FAILED STANDARD THERAPY, IS PREDICTED BY OLDER AGE AND LOWER
} IMMUNOGLOBULIN G AND INR LEVELS

\section{Short running title:}

Age, IgG and INR predict response to MMF in AlH

$\underline{\text { Amanda J Nicoll }}^{1,2}$, Stuart K. Roberts ${ }^{3}$, Ricky Lim ${ }^{4}$, Joanne Mitchell ${ }^{3}$, Martin Weltman ${ }^{4}$, Jacob George $^{4}$, Alan Wigg ${ }^{5}$, Katherine Stuart ${ }^{6}$, Paul Gow $^{3}$, Gerry MacQuillan ${ }^{7}$, Edmund Tse ${ }^{5}$, Miriam Levy ${ }^{4}$, Siddharth Sood ${ }^{3}$, Amany Zekry ${ }^{4}$, Wendy Cheng ${ }^{7}$, Jonathan Mitchell ${ }^{8}$, Richard Skoien ${ }^{6}$, William Sievert ${ }^{3}$, Simone IStrasser ${ }^{4}$, and Geoffrey W McCaughan ${ }^{4}$ on behalf of the ALA Clinical Research Network, Gastroenterological Society of Australia.

${ }^{1}$ Gastroenterology Department, Eastern Health, and ${ }^{2}$ Monash University, Melbourne, VIC;

${ }^{3}$ Melbourne, VIC; ${ }^{4}$ Sydney, NSW; ${ }^{5}$ Adelaide, SA; ${ }^{6}$ Brisbane, QLD; ${ }^{7}$ Perth, WA; ${ }^{8}$ Nambour, QLD.

\section{Authorship:}

This is the author manuscript accepted for publication and has undergone full peer review but has not been through the copyediting, typesetting, pagination and proofreading process, which may lead to differences between this version and the Version of Record. Please cite this article as doi: $\underline{10.1111 / \text { APT.15248 }}$

This article is protected by copyright. All rights reserved 
All authors approved the final version of this manuscript. Guarantor of Article: Dr AJ Nicoll. Manuscript drafting: AJN, SKR, SIS. Manuscript editing and data collection: all authors. Data analysis: SKR, JM, RL. Design of study: SKR, AJN, SIS, GM.

Acknowledgements: This work has been presented as an abstract and poster at Digestive Diseases Week in Washington DC, USA, June $4^{\text {th }} 2018$, and as an oral presentation at Australian Gastroenterology Week, Brisbane, Australia, $8^{\text {th }}$ September 2018.

Declaration of personal and funding interests: None

Conflict of Interest Statement: The authors declare that there are no conflicts of interest pertinent to this work.

Corresponding Author: Professor Amanda Nicoll

Director, Department of Gastroenterology, Eastern Health, Level 3, 3West, 8 Arnold Street, BOX HILL VIC 3128, Australia

Ph: 61-3-9094 9555

Fax: 61-3-9982 2159

Email: amanda.nicoll@easternhealth.org.au

\section{Abbreviations:}

AlH: Autoimmune hepatitis

AHHG: Autoimmune hepatitis group

ALACRN: Australian Liver Association Clinical Research Network

ALT: Alanine aminotransferase

AST: Aspartate aminotransferase

EP: Child-pugh

IgG: Immunoglobulin G

IQR: Interquartile range

MMF: Mycophenolate mofetil

Key words: Autoimmune hepatitis, immunotherapy, mycophenolate mofetil

\section{Abstract:}

This article is protected by copyright. All rights reserved 
Background: Mycophenolate mofetil is a commonly used salvage therapy for patients with autoimmune hepatitis.

Aim: The aim of this study was to evaluate the predictors of response to mycophenolate rescue therapy to facilitate clinical decision making.

Methods: We performed a retrospective observational cohort study of autoimmune hepatitis patients managed in 17 major Australian liver centres who received mycophenolate after an inadequate response or intolerance to corticosteroids with/without thiopurine(s). Baseline demographic, clinical and laboratory variables were compared between responders and nonresponders. A multivariable logistic regression model was developed using forward selection to identify independent predictors of treatment response.

Results: A total of 105 patients received mycophenolate rescue therapy of whom 63 (60\%) achieved biochemical remission. On univariable analysis, older age $(p=0.003)$, INR $<1.1 \quad(p=0.02)$, ethnicity $(p=0.01)$ and lower immunoglobulin gamma $(p<0.002)$ levels were associated with treatment response, while no association was found with cirrhosis status $(p=0.07)$ or treatment indication $(p=0.63)$. On multivariable analysis, lower pre-treatment serum immunoglobulin gamma level $(p=0.01)$, higher age at commencing mycophenolate $(p=0.01)$ and higher INR $(p=0.03)$ were the only significant independent predictors. An immunoglobulin gamma level $<17 \mathrm{~g} / \mathrm{L}$ had a positive and negative predictive value for response of $71 \%$ and $60 \%$ respectively, while age $\geq 54$ years when commencing mycophenolate had a positive and negative predictive value for response of $80 \%$ and $59 \%$, respectively.

Conclusion: Mycophenolate remains an excellent treatment option for patients with autoimmune hepatitis refractory to or intolerant of standard therapy with those most likely to benefit being older and/or having lower pre-treatment immunoglobulin gamma levels. Introduction

Autoimmune hepatitis (AIH) is an incompletely understood inflammatory liver disease that occurs due to loss of tolerance to self-antigens ${ }^{1,2}$. It is characterised by its female predominance, raised transaminases, and association with other autoimmune diseases ${ }^{3}$. Usually AlH responds well to corticosteroids either as monotherapy or in combination with thiopurine(s), with the elevated transaminases and gamma globulins returning to within the normal range over weeks to months and between $75-80 \%$ of patients achieving remission within 2 years ${ }^{4,5}$. A recent review of all randomised controlled trials of AlH therapies, concluded that both corticosteroid monotherapy and corticosteroids in combination with azathioprine were equally effective in inducing remission ${ }^{6}$. However, all 11 studies included in this systematic review were performed before the introduction in 2010 of more stringent criteria to define remission ${ }^{4}$. Subsequently, it has been shown that when 
the 2010 response criteria are applied as opposed to the previous 2002 criteria $^{7}$, the remission rate on standard therapy may be as low as $26 \%^{8}$. Moreover, two-thirds of patients receiving corticosteroid therapy experience significant side-effects including diabetes, hypertension, cataracts, osteoporosis, vertebral compression fractures and psychoses that warrant premature to discontinuation of treatment in up to $13 \%$ of patients ${ }^{9}$. In addition, as many as $25 \%$ of patients on azathioprine develop side-effects including skin rash, fever, arthralgias, nausea, vomiting, pancreatitis and marrow suppression that leads to discontinuation of treatment in about $10 \%$ of patients ${ }^{10}$. Further, maintenance therapy is required in the majority of patients to control inflammation and/or prevent relapse and in the long term, complications of liver disease ${ }^{11}$; this is usually with a thiopurine, with or without a low dose of corticosteroid ${ }^{4,12-14}$. Life expectancy of patients who respond well to therapy is similar to the general population ${ }^{15}$, and response to treatment is the best guide to prognosis ${ }^{8}$.

For patients who fail to achieve a satisfactory response and/or who are intolerant of standard therapy with steroids with/without thiopurine(s), MMF is the most widely used second-line agent in the real-world setting ${ }^{12,16}$. We and others ${ }^{9,17-21}$ have previously shown that treatment with MMF as rescue therapy for AIH appears to be well tolerated and is moderately effective in achieving an overall remission rate of $60 \%$. However, response rates appear to be lower in those with cirrhosis ${ }^{17}$, and those receiving MMF for lack of efficacy rather than intolerance to standard therapy ${ }^{9,20,21}$. The aim of this study was therefore to evaluate the predictors of response to MMF in AlH to facilitate clinical decision making when considering MMF as second line therapy.

\section{Methods:}

The Australian Liver Association Clinical Research Network performed a retrospective, multicentre, observational cohort study of patients with AlH refractory to or intolerant of standard therapy who received MMF as salvage therapy. The study design has been published in detail previously ${ }^{17}$. Seventeen major liver centres across Australia contributed cases, and records were reviewed for demographics, clinical and laboratory characteristics, initial therapy, and treatment outcome. All except one of the 17 liver centres involved in the study were affiliated with a large metropolitan hospital and University, while one site was based in a large regional hospital affiliated with a University. Sites were selected to participate in the study based on an affirmative response by site principal investigators to an expression of interest questionnaire and protocol circulated via email 
to 21 major centres across mainland Australia. Inclusion criteria were age greater than 18 years, a definite or probable diagnosis of $\mathrm{AlH}^{22}$, and having received at least one MMF dose, as shown in Figure 1. Patients with overlap syndromes were excluded, as were those with other concomitant chronic liver disease, human immunodeficiency virus infection, co-existing immunological disorders requiring systemic therapy, active malignancy (except non-melanoma skin cancer) or prior liver transplantation ${ }^{17}$.

The definition of complete response to MMF was alanine transaminase (ALT), aspartate transaminase, and immunoglobulin gamma ( $\lg G$ ) levels returning to the normal reference range, with or without normal liver histology, within the first two years of treatment as previously detailed $^{4}$. Baseline was taken as the results closest to the time of failure of standard therapy and institution of MMF therapy.

The predictors of response in the patients' baseline demographic, clinical and laboratory characteristics were determined by comparing patients who achieved a complete response to MMF to those with no or incomplete response to MMF second-line therapy.

\section{Ethics:}

Cases were de-identified using a unique code including institution, patient's initials, and date of birth. A password-protected electronic database was used to store data. Ethics approval for the study was granted at all sites by the relevant institution's Human Research Ethics Committee.

\section{Statistics:}

Descriptive statistics of the cohort were performed with continuous variables assessed for normality and expressed as mean \pm standard deviation (SD) or median inter-quartile range (IQR) depending on the underlying data distribution. Independent student t-test and Wilcoxon rank-sum test were used where appropriate. Categorical variables were expressed as numbers with percentages. Pearson chi-squared test was used for independent categorical variables. Odds ratios were calculated as effect size, with $95 \%$ confidence intervals $(95 \% \mathrm{Cl})$. Univariable and multivariable logistic regression analyses were performed to determine associations with treatment response and baseline characteristics. Multiple logistic regression was performed using purposeful forward selection methodology as described ${ }^{23}$ using a criterion for inclusion of $p<0.20$ of co-variables on univariable analysis. Where there was high co-linearity between variables, only the variable with the strongest association with treatment response was selected in the model. All 
reported $\mathrm{p}$ values are two-tailed and $\mathrm{p}<0.05$ indicated statistical significance. Analyses were performed with Stata software version 14.1 (StataCorp ${ }^{\circledR}$, College Station, TX, USA).

\section{$\underline{\text { Results: }}$}

\section{Study Population:}

One hundred and five patients met the inclusion and exclusion criteria. The baseline characteristics of the overall cohort are shown in Table 1. The median age at diagnosis of AlH was 50 years (IQR 38-57), and age at starting MMF was 53 years (IQR 43-61), 88\% were female, 86\% were Caucasian, and $97 \%$ were Type $1 \mathrm{AlH}$. Thirty-seven percent of the cohort had cirrhosis, which was established in patients either by liver biopsy and/or on the basis of results of clinical, laboratory and imaging studies ${ }^{24}$. The vast majority (82\%) of these had compensated Child-Pugh A cirrhosis. Previous standard therapy was the combination of corticosteroids (prednisolone and/or budesonide) plus a thiopurine(s) (azathioprine or 6-mercaptopurine) in 98\% of patients. Forty-two (40\%) patients required MMF second-line therapy for refractory disease while $63(60 \%)$ received MMF for treatment intolerance. The main causes of treatment intolerance to azathioprine/6mercaptopurine included: nausea and/or vomiting $(n=20)$ and other gastrointestinal symptoms $(n=4)$, hepatotoxicity $(n=13)$, joint, muscle and/or body pain $(n=7)$, pancreatitis $(n=3)$, fever $(n=3)$, headache $(n=3)$, hair loss $(n=3)$, rash $(n=3)$, bone marrow suppression $(n=2)$, allergic reaction $(n=1)$ and vasculitis $(n=1)$. Two patients were intolerant to prednisolone due to insomnia. Five patients had received treatment with a calcineurin inhibitor (cyclosporin $A, n=2$; tacrolimus, $n=3$ ) a median of 62 months (range: 6.0-177 months) prior to the addition to MMF for reasons of lack of efficacy $(n=3)$ or intolerance to standard therapy $(n=2)$. The median starting and maximum dose of cyclosporin A was $325 \mathrm{mg} /$ day (range $50-600 \mathrm{mg} /$ day) and $350 \mathrm{mg} / \mathrm{d}$ (range: 100-600 mg/day) respectively, and for tacrolimus was $2 \mathrm{mg} /$ day (range: $1-2 \mathrm{mg} /$ day) and $2 \mathrm{mg} /$ day (range: 2-8 $\mathrm{mg} /$ day) respectively. In addition, six patients commenced a calcineurin inhibitor a median of 6.4 months (range: 2.0 to 25.3 months) after commencing MMF due to lack of efficacy ( $n=5$ ) or intolerance $(n=1)$ to MMF. The median and maximum dose of MMF administered was $1 \mathrm{~g} /$ day (IQR 0.88-1.0 g/d) and $2.0 \mathrm{~g} /$ day (IQR $1.0-2.0 \mathrm{~g} /$ day) respectively, while the median duration of therapy was 25 months (IQR 13-60 months).

\section{Comparison of responders and non-responders to MMF:}

Overall, $63(60 \%)$ patients achieved biochemical remission on MMF after a median treatment duration of 12 weeks. This included $3(60 \%)$ of the 5 patients commenced on MMF after prior calcineurin inhibitor treatment including 2 of 3 (67\%) patients receiving calcineurin inhibitor This article is protected by copyright. All rights reserved 
therapy for lack of efficacy and 1 of 2 (50\%) patients treated for intolerance to standard therapy (Table 1). Of note, none of the six patients commenced on a calcineurin inhibitor after MMF nonresponse or intolerance achieved remission. The baseline characteristics of responders and nonresponders are shown in Table 1. Patients who responded to MMF were significantly older at the time of diagnosis of $\mathrm{AlH}$ and at commencing MMF and had a higher frequency of being Caucasian. In addition, responders to MMF had significantly lower serum aminotransferase (i.e. aspartate transaminase, $A L T)$, total bilirubin, immunoglobulin $G(\operatorname{IgG})$ and INR levels than non-responders. The dosing details and indication for treatment with MMF were similar between responders and non-responders, while there was a trend towards a higher frequency of cirrhosis in nonresponders $(p=0.07)$.

\section{Predictors of response in overall cohort}

On univariable analysis, the baseline characteristics that predicted response to MMF included lower IgG levels $(<17 \mathrm{~g} / \mathrm{L})(p=0.002)$, older age ( $\geq 54$ years) at commencing MMF $(p=0.001)$, and lower international normalised ratio $(\leq 1.1)$ (INR) $(p=0.02)$ (Table 2$)$. Cirrhosis was weakly associated with a poorer response to $\mathrm{MMF}(p=0.07)$. There was no association between response to MMF and gender, treatment indication for MMF, and baseline ALT, aspartate transaminase, bilirubin, albumin, creatinine, haemoglobin or platelet levels.

On multivariable analysis, higher age at commencing MMF, lower pre-treatment serum IgG level and lower INR were the only significant independent predictors of a beneficial response to MMF (Table 2). A higher age $\geq 54$ years at the time of commencing MMF had a good positive predictive value of response of $80 \%$ and modest negative predictive value of $59 \%$, while the sensitivity and specificity were $65 \%$ and $76 \%$ respectively. Similarly, serum IgG $<17 \mathrm{~g} / \mathrm{L}$ prior to commencement of MMF had a good positive predictive value for response of $71 \%$ and modest negative predictive value of $60 \%$, while the sensitivity and specificity were $66 \%$ and $72 \%$, respectively. A lower INR of $\leq 1.1$ was also a predictor of good response to MMF however, the clinical significance of the lower INR is unclear as the majority of readings were within normal range in this cohort. Importantly, results of univariable and multivariable analyses were similar after the five patients who received calcineurin inhibitor treatment in combination with standard therapy were excluded. In particular, lower baseline IgG $(<17 \mathrm{~g} / \mathrm{L})$ levels $(p=0.015)$ and older age $(\geq 54$ years) $(p=0.015)$ at the time of commencing MMF remained independent predictors of response while a lower INR $(\leq 1.1)(p=0.08)$ showed a trend towards having predictive value. 


\section{Predictors of response according to treatment indication}

Table 3 shows the results of univariable analysis of the predictors of response to MMF according to MMF treatment indication. In patients who commenced MMF for refractoriness to standard therapy $(n=42)$, only older age at commencing MMF $(p=0.005)$ predicted good response to second-line therapy. In those intolerant to standard therapy $(n=63)$, lower baseline total IgG < 17 $g / L(p=0.008)$, lower INR ( $p=0.03)$, and older age at commencing MMF $(p=0.007)$ were associated with complete treatment response.

On multivariable analysis (Table 4) using a cut-off of $p=0.10$ for variable inclusion, in patients commenced on MMF for non-efficacy of standard therapy, the only significant independent predictor of response to MMF was older age at commencing MMF ( $p=0.006)$. In contrast, in those intolerant to standard therapy the only significant independent predictor of response to MMF was a lower baseline IgG level $(p=0.03)$.

\section{Discussion:}

To our knowledge this is the largest study to examine predictors of treatment response in AlH patients receiving MMF as rescue therapy because of intolerance or inefficacy to standard therapy. The main findings were that among the $105 \mathrm{AlH}$ patients receiving MMF, $60 \%$ of whom achieved remission, younger age and higher pre-treatment IgG levels and higher INR were independently associated with a significantly lower likelihood of treatment response. In addition, we found that the relationship between pre-treatment IgG levels and treatment response was most marked among those receiving MMF for intolerance to standard therapy rather than those who had failed previous treatment, whilst age at commencing MMF, was most relevant in predicting response among those treated for inefficacy of standard therapy. Furthermore, we found that those with cirrhosis had similar responses to MMF compared to those without cirrhosis, and that pre-treatment transaminase levels did not predict the response to second line therapy.

The only other large series of AlH patients receiving MMF rescue therapy found a similar response rate of $69 \%{ }^{18}$, and of interest this result was only marginally lower than the $72 \%$ complete response rate reported by Zachou et $\mathrm{al}^{25}$ in their study of MMF as first-line therapy. The study by Efe et al of 121 patients on MMF rescue therapy had a large number of patients with cirrhosis or pre-cirrhosis (75\% prior to starting MMF). Despite this, pre-MMF IgG levels and biochemistry were quite low in their cohort and INR data was not reported in the context of our observation that This article is protected by copyright. All rights reserved 
lower IgG and INR values are associated with a more responsive phenotype ${ }^{18}$. Data was not provided in this study on the predictors of response to MMF rescue therapy to validate our results.

Our finding that patients with higher IgG levels had a poorer response to MMF rescue therapy may reflect a more aggressive disease and/or immunoreactivity in these patients. One of the largest studies of the natural history of $\mathrm{AlH}^{26}$ showed that predictors of remission on standard first-line therapies included HLA haplotype DRB1*04:01, older age, absence of cirrhosis, and absence of soluble liver pancreas antigen. They also found that younger patients were more likely to relapse. However, their study did not examine baseline IgG levels or INR. There is also evidence to show that long term prognosis is associated with histological activity, and it is interesting to speculate that this may correlate with IgG levels as both reflect the severity of liver inflammation ${ }^{27}$. Certainly, untreated AIH with high IgG levels (greater than twice upper limit of normal), confluent necrosis on liver biopsy, and high transaminase levels, has been shown to have a very poor prognosis ${ }^{28-31}$. Similarly, lower IgG levels appear to be predictive of histological resolution ${ }^{32}$, a lower frequency of relapse ${ }^{33-37}$, and were predictive of successful treatment withdrawal ${ }^{38}$. Other studies have shown that older patients are often less symptomatic at presentation, and often biochemically responsive to therapy ${ }^{14,39-42}$, consistent with our finding of better responses in older patients. However, our results need to be interpreted with some caution as the high IgG correlation with good response to MMF was not demonstrated in those with a poor response to standard therapy, only in those intolerant of corticosteroids and/or thiopurines.

Ours is a real-world cohort of AlH patients that includes patients who have a poor response to standard therapy, as well as those intolerant to the side effects. We pooled these two groups as their overall response rate to MMF was the same ${ }^{17}$. However, when we analysed the data separately, there were differences in the factors predictive of response to MMF, with patients intolerant to standard therapy more likely to respond if they had lower IgG levels, and refractory patients more likely to respond if they were older at diagnosis and/or when starting MMF.

Histologic cirrhosis is associated with reduced long-term survival of AlH patients in some ${ }^{26,40,43}$ but not all ${ }^{44,45}$ studies; however, in this large study cirrhosis status did not confer a negative prognosis to treatment response once other potentially confounding factors were taken into account. This result is similar to that of Zachou et al who failed to show any association between the response to MMF as first-line therapy and the presence or absence of cirrhosis ${ }^{25}$, and that of $\mathrm{Ngu}$ et al who found biochemical response rates to conventional therapy were similar between those with and This article is protected by copyright. All rights reserved 
without cirrhosis ${ }^{45}$. Our finding is not surprising given that bilirubin and aminotransferase levels were not predictive of response; however, a lower baseline INR did predict better response even in patients with previously suboptimal treatment efficacy. The clinical implications are that the INR should be taken into consideration when assessing likelihood of treatment response in patients requiring second line therapy ${ }^{46,47}$.

The strengths of this study are its multicentre, real-world design and cohort size, as it is the second largest study to date to report on the outcomes of second line therapies in AlH patients. Realworld studies such as this and the recent study by Dyson and colleagues from the United Kingdom ${ }^{48}$, provide important insights into the management of $\mathrm{AlH}$ across a wide spectrum of hospitals, and in particular the treatment options and results when standard therapy fails; a point highlighted in a recent editorial by Hupa-Breier et al ${ }^{49}$. While most AlH subjects tolerate first-line treatment well, and achieve remission, for the $20 \%$ who do not, it is important to better understand the factors that influence the effectiveness of second line therapies in order to optimise treatment choice and facilitate patient counselling and expectations. Our study has shown that only $60 \%$ of patients receiving $\mathrm{MMF}$ as second line therapy achieve complete remission ${ }^{17}$, highlighting the importance of identifying the best candidates and looking at possible other agents such as calcineurin inhibitors where necessary. This point was emphasised in a recent article by Janmohamed et al who stressed the importance of intensifying efforts towards identifying patients at greatest risk of treatment failure in order to provide rational personalised management for AlH patients at greatest risk of a poor outcome ${ }^{50}$.

We acknowledge that this study has its limitations, as the data have been collected retrospectively, and as a consequence there are missing data in some fields. For example, whilst liver biopsy data were available on more than $80 \%$ of patients at the time of diagnosis, limited histologic data was available for analysis at the time of starting MMF or to document remission. Moreover, this study does not have longer-term outcome data such as changes in liver histology, transient elastography, or serological markers of fibrosis on MMF therapy. Additionally, we did not collect data on the corticosteroid dose or the number of patients who ceased corticosteroids completely while receiving MMF as part of this work. The sub-analysis of intolerant versus refractory patients has limitations in interpretation due to smaller numbers, and we recommend cautious interpretation about the observed differences. In spite of these limitations, this is the first study to elucidate the characteristics of patients most likely to respond to second line MMF therapy.

This article is protected by copyright. All rights reserved 


\section{Conclusions}

In conclusion, the best predictors of poor response to second line therapy with MMF in AlH are a higher total IgG level and higher INR value. MMF remains an excellent treatment option for AIH patients refractory to or intolerant of standard therapy; however, future studies looking at the long term outcomes of these patients are required. Effective treatment alternatives for patients who fail second line therapy remains a high priority.

\section{References:}

1. Liberal R, Longhi MS, Mieli-Vergani G, et al. Pathogenesis of autoimmune hepatitis. Best Pract Res Clin Gastroenterol. 2011;25(6):653-664.

2. Czaja AJ. Review article: next-generation transformative advances in the pathogenesis and management of autoimmune hepatitis. Aliment Pharmacol Ther. 2017;46(10):920-937.

3. Krawitt EL. Autoimmune hepatitis. New Eng J Med. 2006;354(1):54-66.

4. Manns MP, Czaja AJ, Gorham JD, et al. Diagnosis and management of autoimmune hepatitis. Hepatology. 2010; 51(6):2193-2213.

5. Wang Q, Qiu D, Ma X. Early normalisation of aminotransferase predicts complete biochemical remission in autoimmune hepatitis patients. Aliment Pharmacol Ther. 2011;34(1):107-109.

6. Lamers MMH, van Oijen MGH, Pronk M, et al. Treatment options for autoimmune hepatitis: A systematic review of randomized controlled trials. J Hepatol 2010; 53:191-198.

7. Czaja AJ, Freese DK. Diagnosis and treatment of autoimmune hepatitis. Hepatology 2002; 36:47997.

8. Muratori L, Muratori P, Lanzoni G, et al. Application of the 2010 American Association for the Study of Liver Diseases criteria of remission to a cohort of Italian patients with autoimmune hepatitis. Hepatology 2010; 52:1857.

9. Selvarajah V, Montano-Loza AJ, Czaja AJ. Systematic review: managing suboptimal treatment responses in autoimmune hepatitis with conventional and nonstandard drugs. Aliment Pharmacol Ther. 2012; 36(8):691-707.

10. Gleeson D, Heneghan MA. British Society of Gastroenterology (BSG) guidelines for management of autoimmune hepatitis. Gut 2011; 60:1611-1629.

11. Czaja AJ. Autoimmune hepatitis: focusing on treatments other than steroids. Can J Gastroenterol. 2012;26(9):615-620.

12. Liberal R, de Boer YS, Andrade RJ, et al. Expert clinical management of autoimmune hepatitis in the real world. Aliment Pharmacol Ther. 2017; 45:723-732. 
13. Czaja AJ. Difficult treatment decisions in autoimmune hepatitis. World J Gastro. 2010;16(8):934947.

14. Chen J, Eslick GD, Weltman M. Systematic review with meta-analysis: clinical manifestations and management of autoimmune hepatitis in the elderly. Alment Pharmacol Ther. 2014;39(2):117-124.

15. Schmeltzer PA, Russo MW. Clinical narrative: autoimmune hepatitis. Am J Gastro. 2018. 113(7):951-58.

16. Fallatah $\mathrm{HI}, \mathrm{Akbar} \mathrm{HO}$. Mycophenolate mofetil as a rescue therapy for autoimmune hepatitis patients who are no responsive to standard therapy. Expert Rev Gastroenterol Hepatol 2011; 5:51722.

17. Roberts SK, Lim R, Strasser S, et al. Efficacy and Safety of Mycophenolate Mofetil in Patients With Autoimmune Hepatitis and Suboptimal Outcomes After Standard Therapy. Clin Gastro Hepatol. 2018; 16(2):268-277.

18. Efe C, Hagstrom H, Ytting H, et al. Efficacy and Safety of Mycophenolate Mofetil and Tacrolimus as Second-line Therapy for Patients With Autoimmune Hepatitis. Clin. Gastro. Hepatol. 2017; 15(12):1950-1956 e1951.

19. Jothimani D, Cramp ME, Cross TJ. Role of mycophenolate mofetil for the treatment of autoimmune hepatitis-an observational study. J Clin Exp Hepatol. 2014;4(3):221-225.

20. Baven-Pronk AM, Coenraad MJ, van Buuren HR, et al. The role of mycophenolate mofetil in the management of autoimmune hepatitis and overlap syndromes. Aliment Pharmacol Ther. 2011; 34:335-43.

21. Garcia-Buey L, Moreno-Otero R. Mycophenolate mofetil for patients with autoimmune hepatitis and overlap syndromes. Aliment Pharmacol Ther. 2011; 34:682-685.

22. Alvarez F, Berg PA, Bianchi FB, et al. International Autoimmune Hepatitis Group Report: review of criteria for diagnosis of autoimmune hepatitis. J Hepatol. 1999;31(5):929-938.16.

23. Bursac Z, Gauss CH, Williams DK, et al. Purposeful selection of variables in logistic regression. Source Code Biol Med. 2008;3:17.

24. Bellentani S, Tribelli C, Saccoccio G, et al. Prevalence of chronic liver disease in the general population of northern Italy: the Dionysos study. Hepatology 1994; 20:1442-49.

25. Zachou K, Gatselis NK, Arvaniti P, et al. A real-world study focused on the long-term efficacy of mycophenolate mofetil as first-line treatment of autoimmune hepatitis. Aliment Pharmacol Ther. 2016; 43(10):1035-1047.

26. Kirstein MM, Metzler F, Geiger E, et al. Prediction of short- and long-term outcome in patients with autoimmune hepatitis. Hepatology. 2015; 62(5):1524-1535.

27. Dhaliwal HK, Hoeroldt BS, Dube AK, et al. Long-Term Prognostic Significance of Persisting Histological Activity Despite Biochemical Remission in Autoimmune Hepatitis. Am J Gastro. 2015; 110(7):993-999.

This article is protected by copyright. All rights reserved 
28. Lohse AW. Recognizing autoimmune hepatitis: scores help, but no more. J Hepatol. 2011; 54(2):193-194.

29. Yamamoto K, Miyake $\mathrm{Y}$, Ohira H, et al. Prognosis of autoimmune hepatitis showing acute presentation. Hepatol Res. 2013;43(6):630-638.

30. Fujiwara K, Yasui S, Tawada A, et al. Diagnostic value and utility of the simplified International Autoimmune Hepatitis Group criteria in acute-onset autoimmune hepatitis. Liv Internat. 2011; 31(7):1013-1020.

31. Cook GC, Mulligan R, Sherlock S. Controlled prospective trial of corticosteroid therapy in active chronic hepatitis. Q J Med. 1971;40(158):159-185.

32. Luth S, Herkel J, Kanzler S, et al. Serologic markers compared with liver biopsy for monitoring disease activity in autoimmune hepatitis. J Clin Gastroenterol. 2008;42(8):926-930.

33. Verma S, Gunuwan B, Mendler M, et al. Factors predicting relapse and poor outcome in type I autoimmune hepatitis: role of cirrhosis development, patterns of transaminases during remission and plasma cell activity in the liver biopsy. Am J Gastro. 2004; 99(8):1510-1516.

34. Kanzler S, Gerken $\mathrm{G}$, Lohr H, et al. Duration of immunosuppressive therapy in autoimmune hepatitis. Journal of hepatology. 2001;34(2):354-355.

35. Montano-Loza AJ, Carpenter HA, Czaja AJ. Improving the end point of corticosteroid therapy in type 1 autoimmune hepatitis to reduce the frequency of relapse. Am J Gastro. 2007; 102(5):1005-1012.

36. Czaja AJ, Davis GL, Ludwig J, et al. Complete resolution of inflammatory activity following corticosteroid treatment of HBsAg-negative chronic active hepatitis. Hepatology 1984; 4(4):622627.

37. Al-Chalabi T, Heneghan MA. Remission in autoimmune hepatitis: what is it, and can it ever be achieved? Am J Gastro. 2007; 102(5):1013-1015.

38. Hartl J, Ehlken H, Weiler-Normann C, et al. Patient selection based on treatment duration and liver biochemistry increases success rates after treatment withdrawal in autoimmune hepatitis. $J$ Hepatol. 2015; 62(3):642-646.

39. Al-Chalabi T, Boccato S, Portmann BC, et al. Autoimmune hepatitis (AIH) in the elderly: a systematic retrospective analysis of a large group of consecutive patients with definite AlH followed at a tertiary referral centre. J Hepatol. 2006; 45(4):575-583.

40. Feld JJ, Dinh $\mathrm{H}$, Arenovich $\mathrm{T}$, et al. Autoimmune hepatitis: effect of symptoms and cirrhosis on natural history and outcome. Hepatology 2005; 42(1):53-62.

41. Czaja AJ, Carpenter HA. Distinctive clinical phenotype and treatment outcome of type 1 autoimmune hepatitis in the elderly. Hepatology 2006; 43(3):532-538.

42. Granito A, Muratori L, Pappas G. et al. Clinical features of type 1 autoimmune hepatitis in elderly Italian patients. Aliment Pharmacol Ther. 2005; 21(10):1273-1277.

43. Hoeroldt B, McFarlane E, Dube A, et al. Long-term outcomes of patients with autoimmune 
SK,

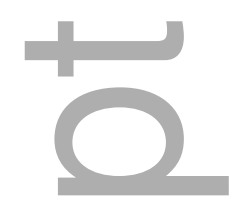

Thernea

u TM,

Czaja AJ.

1

Prognosi

$\circlearrowleft$

s of

histologi

cal

cirrhosis

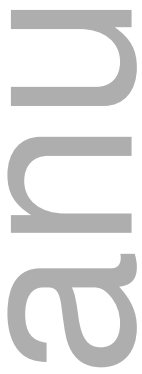

in type 1

autoim

mune

hepatitis

Gastroe

nterology 1996; 110(3):848-857.

45. Ngu JH, Gearry RB, Frampton CM, et al. Predictors of poor outcome in patients with autoimmune hepatitis: a population-based study. Hepatology 2013; 57:2399-2406.

46. Muratori $P$, Carbone $M$, Stangos $G$, et al. Clinical and prognostic implications of acute onset of Autoimmune Hepatitis: An Italian multicentre study. Dig Liver Dis. 2018; 50(7):698-702.

47. Sonthalia N, Rathi PM, Jain SS, et al. Natural history and treatment outcomes of severe autoimmune hepatitis. J Clin Gastroenterol. 2017; 51(6):548-556.

48. Dyson JK, Wong LL, Bigirumurame $\mathrm{T}$, et al. Inequity of care provision and outcome disparity in autoimmune hepatitis in the United Kingdom. Aliment Pharmacol Ther. 2018; 48:951-60.

49. Hupa-Breier KL, Taubert R, Jaeckel E, et al. Editorial: "real-world data" of AlH - time to connect! Aliment Pharmacol Ther. 2018; 48(11-12):1315-16.

50. Janmohamed A, Hirschfield GM. Editorial: autoimmune hepatitis - identifying options for treatment. Aliment Pharmacol Ther. 2016; 43:1232-38.

This article is protected by copyright. All rights reserved 


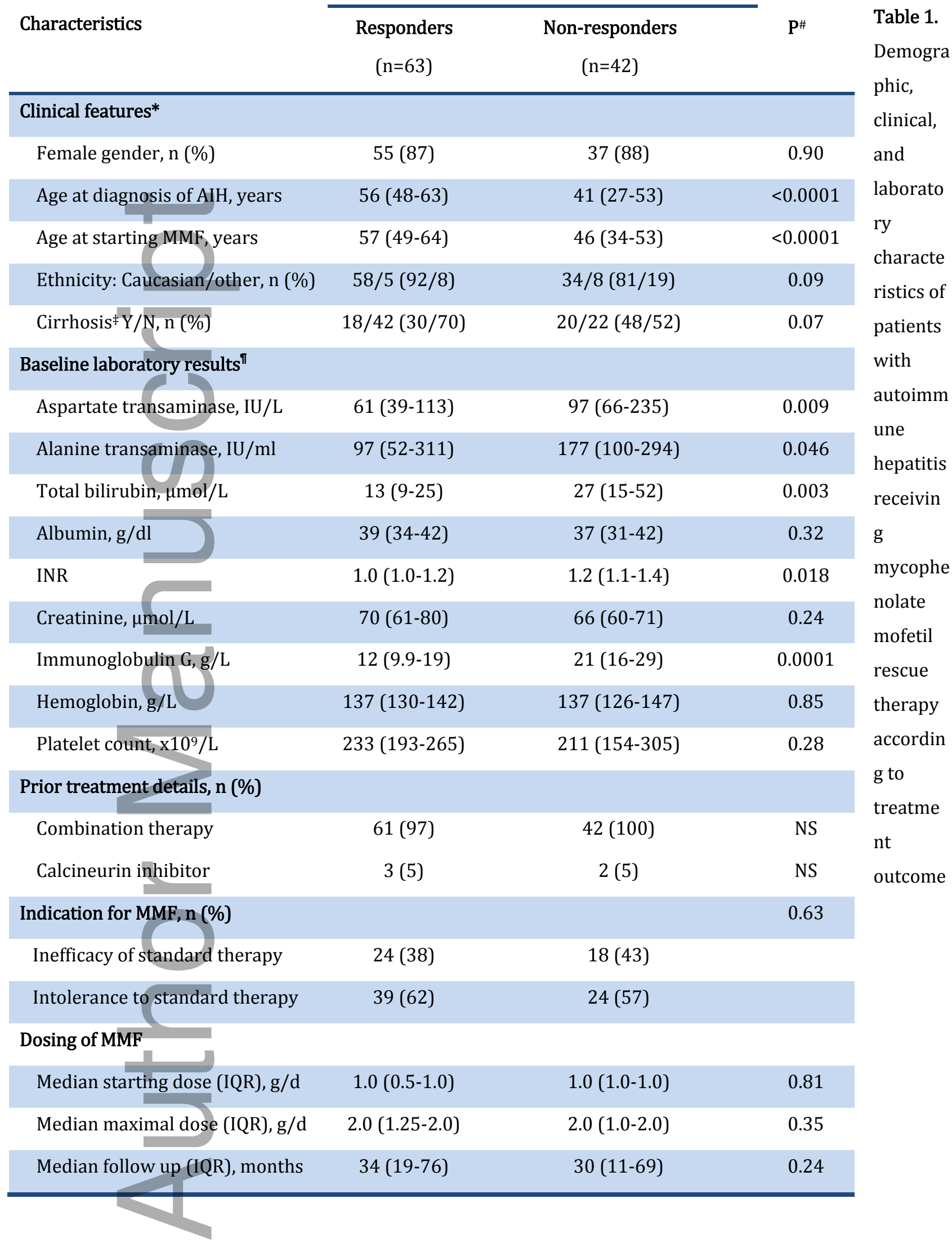




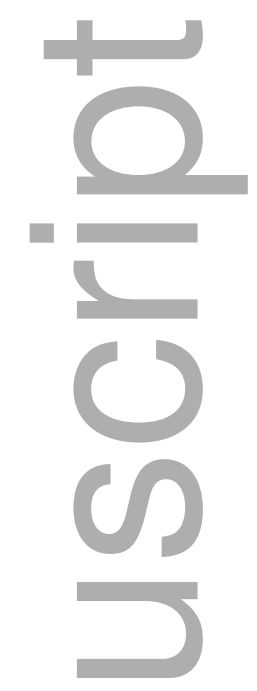

* Results given as median (IQR); IQR=interquartile range

I Baseline values are those immediately prior to starting MMF

$¥$ in 3 patients cirrhosis status was not determined

\# Refers to comparison between responders and non-responders

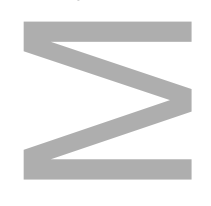

Table 2. Univariable and multivariable analyses of predictors of response to MMF rescue therapy

\begin{tabular}{l|ccc|ccc}
\hline \multicolumn{1}{l}{ Parameter } & \multicolumn{3}{c}{ Univariate analysis } & \multicolumn{3}{c}{ Multivariate analysis } \\
\hline & HR & $95 \%$ CI & P & HR & $95 \%$ CI & P \\
Gender: Male & 1.07 & {$[0.33-3.55]$} & 0.90 & & & \\
& & & & & &
\end{tabular}

This article is protected by copyright. All rights reserved 


\begin{tabular}{|c|c|c|c|c|c|c|}
\hline Age at diagnosis, years & 1.06 & [1.03-1.09] & 0.0001 & & & \\
\hline Age at starting MMF, years & 1.07 & {$[1.03-1.11]$} & 0.0001 & & & \\
\hline Age at starting $M M F ~ \geq 54$ years & 5.96 & {$[2.5-14.4]$} & 0.0001 & 25.3 & {$[2.0-318]$} & 0.01 \\
\hline Ethnicity: Non-Caucasian & 0.37 & {$[0.11-1.21]$} & 0.10 & 0.16 & {$[0.01-1.82]$} & 0.12 \\
\hline Cirrhosis status: Yes & 0.47 & {$[0.21-1.07]$} & 0.07 & 0.35 & {$[0.07-1.65]$} & 0.14 \\
\hline Indication for MMF: Intolerance & 1.22 & {$[0.55-2.7]$} & 0.63 & & & \\
\hline Aspartate transaminase, IU/L & 0.999 & [0.997-1.00] & 0.16 & 1.00 & [0.996-1.01] & 0.77 \\
\hline Total bilirubin, $\mu \mathrm{mol} / \mathrm{L}$ & 0.99 & {$[0.99-1.00]$} & 0.16 & 1.00 & {$[0.97-1.03]$} & 0.98 \\
\hline Albumin, g/dl & 1.04 & {$[0.98-1.11]$} & 0.23 & & & \\
\hline INR level & 0.05 & [0.004-0.72] & 0.03 & & & \\
\hline INR $>1.1$ & 0.27 & {$[0.09-0.79]$} & 0.02 & 0.8 & {$[0.01-0.73]$} & 0.03 \\
\hline Immunoglobulin, $\mathrm{g} / \mathrm{L}$ & 0.91 & {$[0.85-0.97]$} & 0.004 & & & \\
\hline Immunoglobulin $\mathrm{G} \geq 17 \mathrm{~g} / \mathrm{L}$ & 0.20 & {$[0.07-0.56]$} & 0.002 & 0.05 & {$[0.01-0.48]$} & $<0.01$ \\
\hline Creatinine, $\mu \mathrm{mol} / \mathrm{L}$ & 1.01 & {$[0.98-1.04]$} & 0.45 & & & \\
\hline Haemoglobin, g/L & 1.01 & {$[0.97-1.04]$} & 0.63 & & & \\
\hline Platelet count, $\times 10^{9} / \mathrm{L}$ & 1.00 & {$[1.00-1.00]$} & 0.84 & & & \\
\hline
\end{tabular}

NB. All laboratory parameters represent baseline readings just prior to commencing MMF

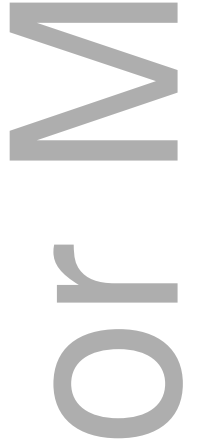

Table 3. Univariable analysis of predictors to response to MMF according to treatment indication

\begin{tabular}{l|ccc|cccc}
\hline & \multicolumn{7}{c}{ Indication for MMF } \\
\cline { 2 - 8 } Parameter & \multicolumn{3}{c}{ Treatment inefficacy } & \multicolumn{3}{c}{ Treatment intolerance } \\
\hline & HR & $95 \%$ CI & P & HR & $95 \%$ CI & P \\
\hline Gender: Male & 2.11 & {$[0.33-3.55]$} & 0.90 & 0.53 & {$[0.11-3.16]$} & 0.53 \\
\hline Age at starting MMF $\geq 54$ years & 11.2 & {$[2.1-60]$} & $\mathbf{0 . 0 0 5}$ & 4.5 & {$[1.5-13.4]$} & 0.007 \\
Ethnicity: Non-Caucasian & 0.70 & {$[0.15-3.28]$} & 0.65 & 0.13 & {$[0.01-1.26]$} & 0.08 \\
\hline Cirrhosis status: Yes & 0.47 & {$[0.13-1.69]$} & 0.25 & 0.48 & {$[0.17-1.40]$} & 0.18 \\
Aspartate transaminase, IU/L & 0.998 & {$[0.995-1.00]$} & 0.18 & 0.999 & {$[0.998-1.00]$} & 0.42
\end{tabular}




\begin{tabular}{l|ccc|ccc} 
Total bilirubin, $\mu \mathrm{mol} / \mathrm{L}$ & 0.99 & {$[0.985-1.00]$} & 0.30 & 0.995 & {$[0.97-1.03]$} & 0.41 \\
Albumin, g/dl & 1.04 & {$[0.94-1.15]$} & 0.43 & 1.04 & {$[0.95-1.13]$} & 0.40 \\
\hline INR $>1.1$ & 0.37 & {$[0.07-1.97]$} & 0.24 & 0.19 & {$[0.04-0.87]$} & 0.03 \\
\hline Immunoglobulin G $\geq 17 \mathrm{~g} / \mathrm{L}$ & 0.32 & {$[0.06-1.60]$} & 0.17 & 0.14 & {$[0.03-0.60]$} & 0.008 \\
\hline Creatinine, $\mu \mathrm{mol} / \mathrm{L}$ & 1.00 & {$[0.96-1.04]$} & 0.95 & 1.02 & {$[0.98-1.07]$} & 0.32 \\
\hline Haemoglobin, g/L & 1.04 & {$[0.99-1.10]$} & 0.10 & 0.98 & {$[0.93-1.03$} & 0.36 \\
\hline Platelet count, $\times 10^{9} / \mathrm{L}$ & 1.00 & {$[0.997-1.01]$} & 0.25 & 0.996 & {$[0.989-1.00]$} & 0.24 \\
\hline
\end{tabular}

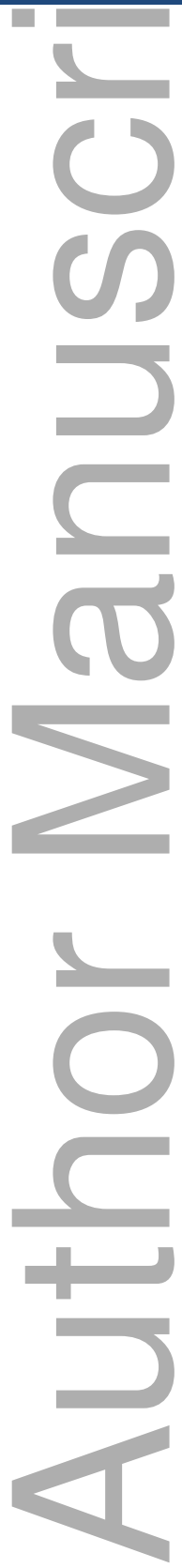


Table 4. Multivariable analysis of predictors to response to MMF according to treatment indication

\begin{tabular}{l|ccc|cccc}
\hline & \multicolumn{7}{c}{ Indication for MMF } \\
\cline { 2 - 7 } Parameter & \multicolumn{3}{c}{ Treatment inefficacy } & \multicolumn{3}{c}{ Treatment intolerance } \\
\hline & HR & $95 \%$ CI & P & HR & $95 \%$ CI & P \\
\hline Age at starting MMF $\geq 54$ years & 11.6 & {$[2.0-68]$} & 0.006 & 12.1 & {$[0.71-205]$} & 0.09 \\
Ethnicity: Non-Caucasian & & & & 0.02 & {$[0.00-1.38]$} & 0.07 \\
INR $>1.1$ & & & & 0.06 & {$[0.003-1.20]$} & 0.07 \\
Immunoglobulin G $\geq 17$ g/L & & & & 0.04 & {$[0.002-0.71]$} & 0.03 \\
Haemoglobin, g/L & 1.05 & {$[0.99-1.11]$} & 0.10 & & & \\
\hline
\end{tabular}

Figure 1: Flow chart showing the patient cohort, exclusions and subgroups.

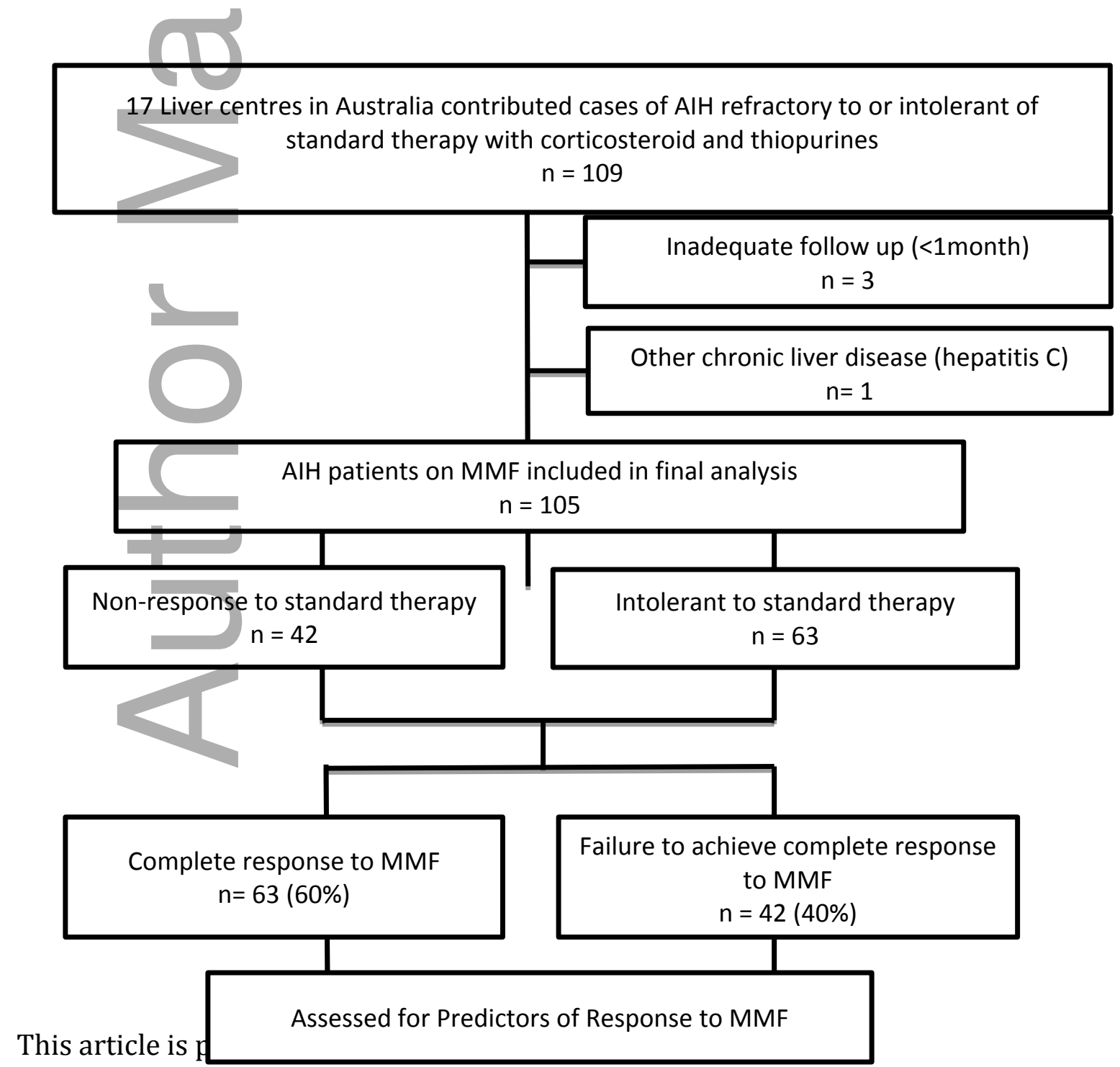




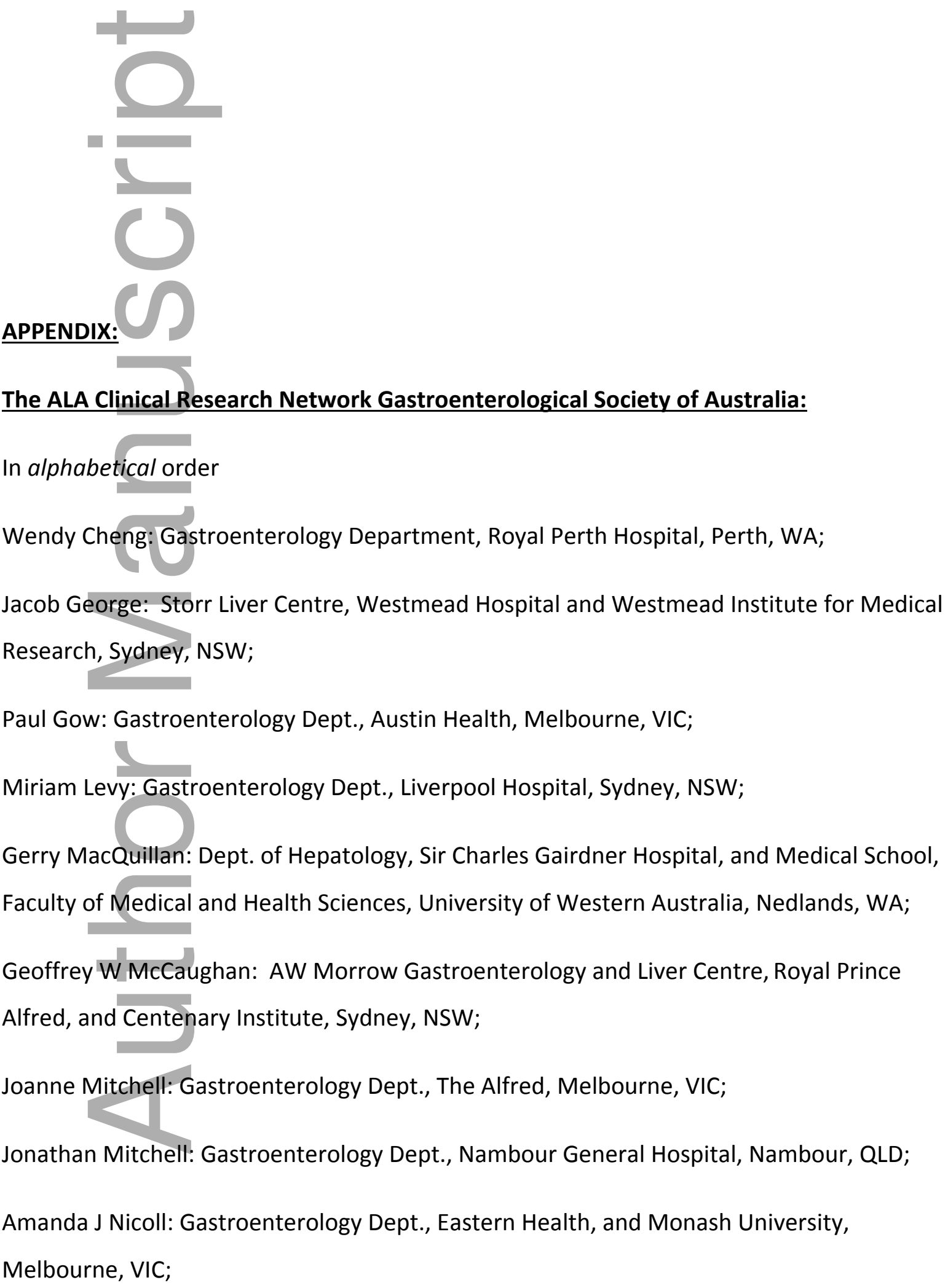

Melbourne, VIC;

This article is protected by copyright. All rights reserved 
Stuart K. Roberts: Gastroenterology Dept., The Alfred, and Monash University, Melbourne, VIC;

William Sievert: Gastroenterology Dept., Monash Health, and Monash University, Melbourne, VIC;

Richard Skoien: Gastroenterology Dept., Royal Brisbane Hospital, Brisbane, QLD

Siddharth Sood: Gastroenterology and Hepatology Dept., The Royal Melbourne Hospital, Melbourne, VIC

Simone I Strasser: AW Morrow Gastroenterology and Liver Centre, Royal Prince Alfred, and The University of Sydney, Sydney, NSW;

Katherine Stuart: Gastroenterology Dept., Princess Alexandra Hospital, Brisbane, QLD;

Edmund Tse: Gastroenterology Dept., Royal Adelaide Hospital, Adelaide, SA;

Martin Weltman: Gastroenterology Dept., Nepean Hospital, NSW;

Alan Wigg: Dept. Gastroenterology and Hepatology, Flinders Medical Centre, Adelaide, SA; Amany Zekry: Dept. Gastroenterology, St George Hospital, Sydney, and Dept. of Medicine, University of New South Wales, Sydney, NSW

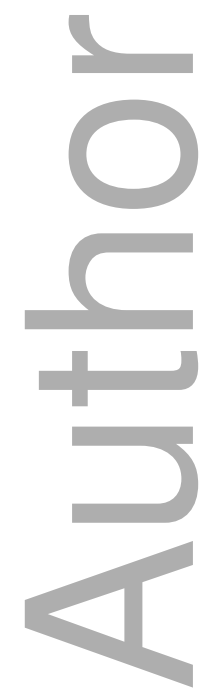




\section{University Library}

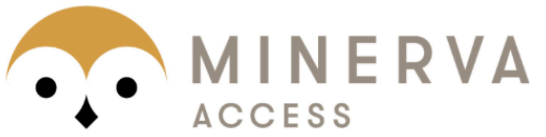

A gateway to Melbourne's research publications

Minerva Access is the Institutional Repository of The University of Melbourne

\section{Author/s:}

Nicoll, AJ;Roberts, SK;Lim, R;Mitchell, J;Weltman, M;George, J;Wigg, A;Stuart, K;Gow, P;MacQuillan, G;Tse, E;Levy, M;Sood, S;Zekry, A;Cheng, W;Mitchell, J;Skoien, R;Sievert, W;Strasser, SI;McCaughan, GW;Cheng, W;George, J;Gow, P;Levy, M;McCaughan, GW;Mitchell, J;Mitchell, J;Nicoll, AJ;Roberts, SK;Sievert, W;Skoien, R;Sood, S;Strasser, SI;Stuart, K;Tse, E;Weltman, M;Wigg, A;Zekry, A

Title:

Beneficial response to mycophenolate mofetil by patients with autoimmune hepatitis who have failed standard therapy, is predicted by older age and lower immunoglobulin $\mathrm{G}$ and INR levels

\section{Date:}

2019-05-01

\section{Citation:}

Nicoll, A. J., Roberts, S. K., Lim, R., Mitchell, J., Weltman, M., George, J., Wigg, A., Stuart, K., Gow, P., MacQuillan, G., Tse, E., Levy, M., Sood, S., Zekry, A., Cheng, W., Mitchell, J., Skoien, R., Sievert, W., Strasser, S. I. ,... Zekry, A. (2019). Beneficial response to mycophenolate mofetil by patients with autoimmune hepatitis who have failed standard therapy, is predicted by older age and lower immunoglobulin G and INR levels. ALIMENTARY PHARMACOLOGY \& THERAPEUTICS, 49 (10), pp.1314-1322. https:// doi.org/10.1111/apt.15248.

Persistent Link:

http://hdl.handle.net/11343/285727 